\title{
The Application of IPv6 Technology in Several Typical Industries and Its Application Innovation in the Public Security Industry
}

\author{
Fuyu Tao*, Pengchao Li \\ Chongqing Police College, Chongqing, China \\ Email: *1348962889@qq.com
}

How to cite this paper: Tao, F.Y. and Li, P.C. (2022) The Application of IPv6 Technology in Several Typical Industries and Its Application Innovation in the Public Security Industry. Advances in Internet of Things, $12,1-8$.

https://doi.org/10.4236/ait.2022.121001

Received: November 17, 2021

Accepted: December 12, 2021

Published: December 15, 2021

Copyright $\odot 2022$ by author(s) and Scientific Research Publishing Inc. This work is licensed under the Creative Commons Attribution International License (CC BY 4.0).

http://creativecommons.org/licenses/by/4.0/

\section{(c) (i) Open Access}

\begin{abstract}
This paper discusses the advantages of IPv6 technology compared with IPv4, such as address capacity, automatic address allocation, mobile connection characteristics, security and confidentiality, quality of service, and multicast transmission. The key summary summarizes the application of IPv6 technology in the four industries of power, finance, home, and education. Since there is almost no application of IPv6 technology in the public security industry in the previous literature, the author proposes the application innovation of IPv6 technology in the public security industry according to his own industry. IPv6 technology can be applied to the work communication, information exchange, case investigation, and social security management of public security departments, which helps to improve the level of public security work, the quality of the people's police, and the efficiency of public security affairs, thereby improving the safety and satisfaction of the general public. Spend. It has a certain reference significance to make a great contribution to reducing the crime rate, creating a harmonious social environment, and truly realizing the integration of police and civilians.
\end{abstract}

\section{Keywords}

IPv6, Technical Advantages, Industry Applications

\section{Introduction}

In recent years, with the rapid development of Internet commercialization, the large-scale expansion of the Internet, and the increasing demand for new network applications, the development of the Internet has begun to face numerous challenges. The more tricky ones are: the upper limit of bandwidth is restricted, 
the network has many security loopholes, the supervision of the Internet is more difficult, the address space is relatively scarce, the quality of service is difficult to guarantee, etc. [1]. However, the Internet protocol IPv4, which played a key role in the development of the Internet and is widely used in various underlying networks, has become increasingly tense. The application of the Internet is restricted due to the inability to meet the needs of the majority of users. At the same time, it has delayed the rapid development of the Internet [2]. In order to eradicate the potential crisis of IPv4 network address exhaustion, the Internet Engineer Task Force (IETF) proposed the next-generation Internet protocol IPv6 to solve the many problems encountered before [3].

At present, IPv6 technology has not disappointed us. It is widely used in many representative industries such as electricity, finance, households, education, and military, and has achieved exciting results. Nowadays, the diversification and technicalization of illegal and criminal methods in society are emerging. Public security departments must also use emerging scientific and technological means to combat illegal and criminal activities. Therefore, the application innovation of IPv6 technology in the public security industry is proposed. Research shows that IPv6 technology can assist the public security system to play an important role in cracking down on crimes and solving many problems that were difficult to solve before.

\section{Advantages of IPv6 Technology}

- IPv6 has a larger address space than IPv4. The length of IP address in IPv4 is 32 , that is, the maximum number of addresses is $2^{32}$. The length of IP address in IPv6 is 128 , that is, the maximum number of addresses is $2^{128}$. Compared with the former, the address space of IPv6 is 296 times larger.

- The routing table used by IPv6 is smaller, and IPv6 address allocation directly follows the principle of clustering. Therefore, the router can represent a subnet with only one record in the routing table, thereby reducing the length of the routing table and speeding up the packet forwarding speed of the router.

- IPv6 adds new flow control and enhancements to multicast support, which provides long-term development opportunities for multimedia applications on the network and solves the difficult problem of service quality mentioned above.

- IPv6 supports automatic configuration, which makes it easier to manage the network, especially the LAN.

- The security of IPv6 is greatly improved. When users are using IPv6 networks, they can encrypt the data in the network layer and verify the IP messages. The authentication and encryption options in IPv6 provide packet integrity and confidentiality, and the network security is greatly increased.

- IPv6 allows the protocol to be expanded in the face of new technologies and new application requirements.

- IPv6 adopts a new header format, which separates options from the basic header, and inserts options between the upper-layer data and the basic header when needed. This can simplify and speed up the router selection process and 
avoid the waste caused by most options that do not require routing.

- IPv6 has added some new options to implement additional functions [4].

Based on the many advantages of IPv6 mentioned above, in the third section, we take IPv6 in the four industries of power, finance, household, and education as examples to summarize in detail the application and important role of IPv6 in these four industries.

\section{Application of IPv6 Technology in Many Industries}

\subsection{Application in the Power Industry}

\subsubsection{Enhancement of Information and Communication Network Capabilities}

Use IPv6 technology to optimize and improve the current communication network, provide a strong guarantee for the transmission of real-time control services of the power grid, meet the rapidly growing demand for high-bandwidth IP services, and improve the integrated control and security capabilities of the power information communication network, business support capabilities, business carrying capabilities and business service capabilities.

\subsubsection{In Terms of Terminal Access}

Due to the wide coverage of smart grid construction, involving various types of detection, sensors and other terminal equipment, there is a high demand for address space access capabilities. IPV6 can well overcome the problem of terminal equipment IP address resource shortage. And IPv6 can ensure faster and safer transmission.

\subsubsection{In terms of Mass Storage}

The use of sensors and radio frequency identification (RFID) tags and other devices has rapidly increased the demand for IP addresses, and cloud computing also plays an important role in the storage and processing of mass data. Therefore, IPv6 can meet the storage capacity of massive information.

\subsubsection{In Terms of Intelligent Control}

IPv6 technology can perform grid-connected control of distributed energy and new energy, orderly use electricity management, promote business melting and information sharing, and achieve real-time line loss and other goals.

\subsubsection{In Terms of Information Security Protection}

IPv6 can provide more and stronger security protection, while speeding up forwarding, strengthening information security protection, enabling information security to be autonomous and controllable, preventing smart terminals from being invaded and controlled, and preventing business data. The information is maliciously tampered with to prevent the network from being maliciously monitored.

\subsubsection{In Terms of Improving Service Quality}

IPv6 technology can improve the efficiency of information and communication 
network in the power Internet, provide a strong guarantee for the business continuity of future IPv6 users, and at the same time improve the service quality of the power grid to achieve the purpose of improving the quality of the smart grid [5].

\subsection{Application in the Financial Industry}

\subsubsection{Changes in Network Architecture}

At present, all current basic grid architectures are based on IPv4. With the wide application of virtual local area network (VALN) technology and virtual private network (VPN) technology, private IPv4 addresses are reused. Many problems such as too complicated routing planning and high difficulty in operation and maintenance management have been exposed. IPv6 technology has the characteristic of "each grain of sand has its own IP address", so it can bring huge optimization to network planning and design, routing protocol planning and design, security, and operation and maintenance management. Under the application of IPv6 technology, each device can be interconnected through a unique address in the backbone network, extranet and metropolitan area network, and there is no need to convert routing protocols, and the devices can communicate directly, which greatly enhances the network Security, while improving the routing efficiency and speed.

\subsubsection{Breakthrough in Video Applications}

IPv6 and the router addressing method that has improved the traditional IPv4 will undoubtedly greatly enhance the user experience of video services. IPv6 solves the problem of address capacity that has always existed before, improves the address structure, effectively improves the data throughput and routing efficiency, and satisfies the demand for the large amount of information required for video transmission. IPv6 technology uses technologies such as multicast and multicast to effectively utilize existing network bandwidth, creating conditions for multi-user video conferencing and ultra-definition television broadcasting. IPv6 improves the security of end-to-end transmission, improves service quality, reduces the pressure of system management, and pushes Voice over Internet Protocol (VoIP), online games, and mobile Internet to a new level. At the same time, in the IPv6 environment, due to its simplified network addressing and the addition of anycast, the use of network traffic and bandwidth will be reduced, which improves the real-time transmission of data.

\subsubsection{The Rapid Development of Financial Services}

In the IPv6 network environment, mobile devices such as mobile phones and tablet computers can quickly and efficiently access the website, which is easy to expand the user group and make it convenient for the majority of user groups to use financial services. Especially with the introduction of mobile communication systems from generation to generation, mobile IPv6 services will definitely be the general direction for the development of mobile financial services in the fu- 
ture, and the IPv6 protocol will carry mobile communications and the Internet. The large-capacity address space of IPv6 can ensure that every mobile terminal obtains a globally unique IP address, so that personal tablets, mobile phones and other devices can quickly connect to the network. It can be said that in the IPv6 environment, whether traditional online banking, online securities, electronic transfers, or the well-known mobile payment, third-party payment, etc., will obtain a larger user group and a larger business volume [6].

\subsection{Application of IPv6 in Home Network}

\subsubsection{Remote Monitoring and Control of Household Appliances}

One of the main applications of the home network is the remote control and monitoring of home appliances. Under the home network based on IPv6, it can be used for purposeful surveillance through voice calls or web interface for specific area surveillance, residential security, etc. At the same time, it is possible to understand and control the situation of home appliances through the web interface to prevent a series of disasters caused by abnormal home appliances when there is no one in the home.

\subsubsection{Residential Surveillance System and Family Safety Precautions}

The residential surveillance system is a real-time surveillance system built on the home network infrastructure. It can alert in time when responding to intrusions or discovering abnormal phenomena, and transmit the situation of the crime scene to the user's mobile device through the home network. At the same time, the residential surveillance system can also be combined with door and window sensors, access control systems, etc. to jointly build a protective wall for home security.

\subsubsection{Early Warning in Case of Emergency}

The basic functions of home network applications also include early warning in emergencies. It combines home access control or door and window sensors to face many security problems in the family, such as burglary, gas leakage, electricity leakage, fire, etc., and can promptly warn home users or residential property, and assist them in timely remedy or alarm. Minimize the loss of profits [7].

\subsection{Application of IPv6 in Education Industry}

\subsubsection{Online Teaching Resources Are More Abundant}

Compared with the troubles of network bandwidth and transmission mechanism a few years ago, the educational resources of most domestic websites are mainly static educational resources such as text and pictures, and very few websites will have audio and video educational resources. Far from meeting the needs of large-scale teaching, IPv6 overcomes the problem of addressing capacity barrenness and expands data throughput. This is where we can learn through smooth and clear teaching videos anytime and anywhere. At the same time, IPv6 has strengthened its multicast function, reaching the application of high-definition digital TV, video conferencing, and video-on-demand based on multicast, pro- 
viding students and learners with massive online learning resources.

\subsubsection{Online Distance Teaching}

The previous Internet was unable to achieve large-scale end-to-end audio and video communications due to limited bandwidth. Therefore, online teaching in the early years was more interactive through words and symbols, which greatly affected the quality of online learning. IPv6 technology has changed the original data transmission method and message segmentation method, so that the use of bandwidth can be reduced while transmitting as much data as before, making audio and video widely used on the network. In the current online course learning, teachers and students can interact not only through audio, but also through clear and smooth video, which makes the online courses lively and real, as if they are in a classroom, greatly improving the quality of distance teaching.

\subsubsection{Implement Mobile Learning}

Mobile learning usually refers to learning at any place and at any time with the assistance of mobile devices, and the mobile devices used in mobile learning can also enable teachers and students to have two-way interactive communication. The previous mobile communication technology mainly uses voice Business-oriented, but with the development of society and the continuous improvement of user needs, the new generation of mobile communication systems needs to implement services such as mobile multimedia, real-time voice, and mobile e-commerce. However, the realization of these services is not easy, and it is limited in many aspects such as roaming, terminal, and charging. IPv6 technology can overcome the above-mentioned many problems. IPv6 has a huge address space, guaranteed quality of service, automatic address allocation, good mobility support, and excellent features such as security. Therefore, businesses at home and abroad are accelerating the construction of an all-IP core network based on IPv6. In this way, students can use mobile devices to start learning at any time and anywhere, and are no longer limited by external interference factors such as location and time. It can be said that IPv6 has created good conditions for mobile learning [8].

In this section, we have made a detailed introduction to the application of IPv6 in multiple industries and found that IPv6 technology has accelerated the intelligentization process in these fields. Based on the above advantages, the author found that IPv6 can also be applied to the public security industry in which he is located. In the fourth section, the application innovation of IPv6 technology in the public security industry is proposed.

\section{Application Innovation of IPv6 in Public Security Industry}

\subsection{IPv6 Technology Enables Efficient Work Meetings}

IPv6 meets the demand for the large amount of information required for video transmission, and the technology uses multicast and multicast technologies to effectively utilize the existing network bandwidth. At the same time, in the IPv6 environment, the real-time data transmission performance is excellent, and it 
has the advantages of stability, security, and reliability. Therefore, large-scale high-definition video conferences can be held at any time, which is conducive to the timely arrangement and up-and-down transmission of major tasks by the public security department. Police offices and police stations in various jurisdictions can also conveniently and quickly hold video work conferences between various departments to carry out work reports, research and discussion, and task notifications in a timely manner. This is conducive to the exchange of work between leaders at all levels and speeds up the internal public security system. The deployment of work has enhanced the efficiency of the public security system and made up for the shortcomings of traditional telephone conferences, emails and other communication methods.

\subsection{IPv6 Technology Facilitates Timely Remote Command}

The data transmission based on IPv6 technology has excellent audio and video synchronization and strong mobility. Commanders can clearly observe the images transmitted back from the crime scene just by staying in the command center, and can carry out remote solutions in the first time deployment and command and dispatch to realize the fastest transmission of orders, the optimization of deployment arrangements, and the fastest feedback of front-line and rear information, which solves the difficulty of commanders not being able to arrive at the scene in the first time, and plays a key role in the control of emergencies.

\subsection{IPv6 Technology Can Speed up the Detection of Cases}

At present, the diversification and technicalization of illegal and criminal methods are emerging. The activity, concealment and confrontation of suspects are becoming stronger and stronger. The public security department must adopt new working models and advanced information processing methods to effectively combat illegal crimes. Activities, science and technology strong police is the main trend of future police force improvement. IPv6-based video conferencing systems and surveillance cameras have a qualitative leap in the quality and smoothness of the video. When the suspect has high mobility, the police stations can use remote video to monitor the progress of the case in time. Mutual case assistance, information exchange, and timely sharing of collected images, voices, and videos of criminals, so that different regional departments can assist each other to achieve the purpose of case detection, speed up the progress of case detection, and improve the efficiency of case detection.

\subsection{IPv6 Technology Can Make Public Security Intelligent}

The IPv6 header contains some information about controlling the quality of service (flow marking and flow category). By configuring the router, you can control the priority and guarantee the quality of service, which greatly improves the quality of service, and the excellent quality of service can be guaranteed from VoIP to high-quality transmission of video streams, real-time and efficient social 
security monitoring is completed. At the same time, convenient projects such as online police stations, online police offices, and firefighting online can be established through the internal public security network. Whether illegal and criminal activities are obtained through monitoring or the citizens themselves report to the police through the convenience projects, it will help the public security organs to dispatch police in time. The overall level of public security work, the overall quality of the people's police, and the sense of security and satisfaction of the general public have been greatly improved.

\section{Conclusion}

IPv6 has features such as simplified header format, automatic configuration of host addresses, authentication, encryption, and strong mobile communication support capabilities, which have greatly promoted the intelligentization process in multiple industries such as electricity, finance, households, and education, in order to gain stronger vitality in these areas. This article fully introduces the advantages of IPv6 technology, and summarizes its application in many representative industries, and puts forward the application innovation of IPv6 technology in the public security industry, which has certain reference significance.

\section{Acknowledgements}

Supported by the Science and Technology Research Program of Chongqing Municipal Education Commission (Grant No. KJQN202001709).

\section{Conflicts of Interest}

The authors declare no conflicts of interest regarding the publication of this paper.

\section{References}

[1] Luo, Q.W. (2004) Current Status of IPv6 Development at Home and Abroad. Mobile Communications, 28, 35-38.

[2] Li, Z.D. (2012) Discussion on the Development of IPv6. Computer CD Software and Application, 21, 2.

[3] Cheng, G.L. (2009) IPv6 Application Status and Industry Prospect Analysis. Technology and Industrialization of Chinese Universities, 10, 3.

[4] Chen, X. and Zhao, H. (2013) Theory and Practice of Informatization Construction in Chinese Colleges and Universities. National School of Administration Press, Beijing, 40-41.

[5] Guo, J.H. (2013) Application Prospect of IPv6 in Smart Grid. Electric Power Information Technology, 11, 9-16.

[6] Ye, B.H. (2012) The Application Prospects of IPv6 in the Financial Industry. Financial Electronics, 9, 2.

[7] Zhao, B. (2006) Application of IPv6 in Information Appliances. Journal of Shengli Oilfield Workers' University, 5, 81-82.

[8] Cheng, Z. and Zhou, T. (2005) IPv6 and Its Application in Education and Teaching. China Audio-Visual Education, 9, 97-99. 